\title{
Hyperfine Splitting of Excited States of New Heavy Hadrons and Low-Energy Interaction of Hadronic Dark Matter with Photons, Nucleons, and Leptons
}

\author{
Vladimir Kuksa *,+ (D) and Vitaly Beylin ${ }^{+}$ \\ Research Institute of Physics, Southern Federal University, Av. Stachky 194, Rostov-on-Don 344090, Russia; \\ vitbeylin@gmail.com \\ * Correspondence: vkuksa47@mail.ru \\ + These authors contributed equally to this work.
}

Received: 12 May 2020; Accepted: 18 June 2020; Published: 19 June 2020

check for updates

\begin{abstract}
We consider the structure of excited states and low-energy interaction of hadronic dark matter with photons, leptons, and nucleons. Description of the lowest excited levels is fulfilled in an analogy with the standard heavy-light mesons. Using the effective vertex of new heavy hadrons interaction with $W$-boson, we calculate cross-section of the lepton scattering on the dark matter particle. Analysis of strong low-energy interaction of new hadrons was carried out within the effective meson-exchange model based on dynamical realization of SU(3)-symmetry. A cross-section of nucleon scattering on the hadronic dark matter was also calculated using this model. The most essential phenomenological consequences of the low-energy dark matter interaction with leptons and nucleons are discussed.
\end{abstract}

Keywords: new hadrons; hadronic dark matter; interaction

\section{Introduction}

The most popular candidates for the hidden mass particles are massive neutral particles weakly interacting with the ordinary ones (WIMP). Measurements of WIMP-nucleon scattering cross-section [1] exclude some models of WIMPs. Various alternative scenarios are discussed in a lot of papers, for instance, there is a scenario with strongly interacting new heavy particles (SIMP) [2-6]. In the scenario with the hadronic dark matter (DM), new particles consist of new heavy stable quark and the standard one, $(q Q)$. New quarks arise, for example, in the extension with singlet quark [4], chiral-symmetric models [5,7] and in the Standard Model (SM) extension with the 4th generation [8-10]. Main properties of new hadrons and their low-energy phenomenology were considered in [4-6]. As it was noted in this paper, repulsive asymptotic of the DM particles interaction with nucleons provides an agreement with the strong cosmochemical restrictions on concentrations of anomalous hydrogen and helium [6,7]. In this work, we consider and present theoretical basis which is used for the description of the low-energy phenomenology of hadronic dark matter. The basis is constructed in the framework of the SM extension with singlet quark [4] and chiral-symmetric model [5], where new quarks interact with photon and Z-boson (not with W-boson). Some aspects of high-energy DM-phenomenology, for instance the process of DM annihilation, were considered in review work [6]. Here, we restrict themselves by the qualitative description of the most typical signals of the DM-annihilation process (see Section 4).

Description of the signals generated in the processes with the DM particles participation should be based on the knowledge about features of their interaction with the usual matter. Here, the structure of the lowest excited states of new heavy hadrons is considered, together with the low-energy electro-weak 
and strong interactions of the hadronic DM with photons, leptons, and nucleons. To calculate cross-section of the DM-lepton scattering, an effective Lagrangian of the DM interactions with the gauge bosons was found in the limit of the small momentum transfer. Strong interaction of new hadrons with nucleons is considered in the effective meson-exchange model basing on the dynamical realization of $\mathrm{SU}(3)$-symmetry. Note that use of the effective meson-exchange model is reasonable because the heavy DM is cold and essentially non-relativistic at this time. Validity of this approach is ensured by the fact that momentum transfer is small for the case of nucleon scattering on the cold DM. With the model Lagrangian, for the nucleon scattering on hadronic DM particles, we derived analytical expressions for the cross-section of the process; these cross-sections can be used in the study of the low-energy DM phenomenology.

The paper is organized as follows. In Section 2, we consider the structure of the lowest excited states of new heavy hadrons and possible electromagnetic transitions between them. Effective Lagrangian of the DM interaction with $W$-boson is constructed in Section 3, where the cross-section of DM-electon scattering is also calculated. In Section 4, effective Lagrangian of the DM interactions with mesons at low energies is derived; analytical expressions for the cross-sections of the DM-nucleons scattering are also given here. Discussion and some conclusions are represented in Section 5.

\section{Structure of the Lowest States of New Hadrons}

Some general characteristics of new heavy hadrons, such as mass, lifetime, and energy spectrum of the lowest excited states, can be described analogous with the standard heavy-light mesons. Gross hierarchy of the mass spectrum of new hadrons directly follows from their quark content: $M \rightarrow$ $(q Q),(q q Q) ; 2 M \rightarrow(Q Q),(q Q Q) ; 3 M \rightarrow(Q Q Q)$, where $M$ is the mass of new heavy quark $Q$. Fine structure of this hierarchy is generated by differences of light quarks masses and the binding energies, it is defined by quantum numbers of system $(q Q)$. Quark content and quantum numbers of two- and three-quark heavy hadrons are represented in [4], where the main properties of new heavy mesons were considered.

At first, we consider the lightest neutral and charged pseudoscalar states $\left(J^{P}\right)=\left(0^{-}\right)$with quark composition $M^{0}=(u \bar{U})$ and $M^{-}=(d \bar{U})$. Analogous with ordinary heavy-light mesons, $D$ and $B$, new heavy meson $M$ is the lightest (ground) state of two-quark systems with orbital moment $L=0$, full moment $J=0$ and parity $P=(-1)^{1+L}=-1$ (pseudoscalar state). The vector and scalar $M$-mesons are the excited two-quark states, and furthermore we estimate the value of corresponding mass-splitting. In the case of standard heavy-light mesons, the mass-splitting of charged and neutral component is an order of MeV. Some argumentation was presented in [4] that the value of mass-splitting for new mesons is the same order and $\Delta M=m\left(M^{-}\right)-m\left(M^{0}\right)>m_{e}$. The last inequality follows from the destabilization condition of the charge component that is the charged meson has the decay channel $M^{-} \rightarrow M^{0} e \bar{v}_{e}$. As it was evaluated in [4], due to the fine mass-splitting $\Delta M$, the $M^{-}$lifetime can be large, $\tau \sim\left(1-10^{5}\right) \mathrm{s}$ (at $\Delta M=1-10 \mathrm{MeV}$ ). Thus, the charged component of new heavy hadrons can be metastable. This property makes it possible to detect $M^{-}$or its decay products, low-energy electron and antineutrino pair, provided that $M^{-}$is generated. The process of charged component generation in the $e M^{0}$ collisions is considered in the next section, where the corresponding cross-section of $e M^{0}$ scattering is calculated.

Now, we consider the hyperfine splitting of ground and excited states, $\delta M_{q}=m\left(M_{q}^{*}\right)-m\left(M_{q}\right)$, where $M_{q}^{*}$ is the lowest excited state of $M$-particle. Here, we restrict ourselves by the simplest case of heavy mesons $M_{q}=(q \bar{U})$. Fully analogous with the standard heavy-light mesons, $D_{q}=(c q)$ and $B_{q}=(\bar{b} q)$, we describe the ground and excited states in the terms $S_{0}^{1}$ and $S_{1}^{1}$ (in the classification $\left.L_{J}^{2 s+1}\right)$, or $\frac{1}{2}\left(0^{-}\right)$and $\frac{1}{2}\left(1^{+}\right)$(in the classification $I\left(J^{P}\right)$ ). Here, $L, s, J, I$, and $P=(-1)^{1+L}$ are orbital momentum of system, spin, full momentum, isospin and parity, respectively. The ground states $\frac{1}{2}\left(0^{-}\right)$ of usual and new mesons we will designate as $D_{q}, B_{q}$, and $M_{q}$, while the excited states as $D_{q}^{*}, B_{q}^{*}$, and $M_{q}^{*}$. To evaluate the mass-splitting between heavy states $M_{q}$ and $M_{q}^{*}$, we use an analogy with the splitting mechanism in standard heavy-light mesons, which was formulated in the framework of 
heavy quark effective theory. Heavy quark symmetry provides relations between masses of excited states of $B$ and $D$ mesons [11]:

$$
m\left(B_{2}\right)-m\left(B_{1}\right) \approx \frac{m_{c}}{m_{b}}\left(m\left(D_{2}\right)-m\left(D_{1}\right)\right),
$$

where $m\left(B_{k}\right)$ and $m\left(D_{k}\right)$ are the masses of $B_{k}$ and $D_{k}, m_{c}$, and $m_{b}$ are the masses of constituent quarks. One can check that this relation approximately describes the splitting between first excited $\frac{1}{2}\left(1^{-}\right)$and ground states $\frac{1}{2}\left(0^{-}\right)$of $B$ and $D$ mesons with good accuracy:

$$
\frac{m\left(B^{*}\right)-m(B)}{m\left(D^{*}\right)-m(D)} \approx \frac{m_{c}}{m_{b}} \longrightarrow 0.32 \approx 0.32(0.28) .
$$

In (2), we have used $m\left(B^{*}\right)-m(B)=45 \mathrm{MeV}$ and $m\left(D^{*}\right)-m(D)=142 \mathrm{MeV}$ (see [12]), $m_{c}=1.55 \mathrm{GeV}$ and $m_{b}=4.88 \mathrm{GeV}$ [11]. The value in bracket follows from the data on masses $m_{c}=1.32 \mathrm{GeV}$ and $M_{b}=4.74 \mathrm{GeV}$ which are used in Reference [13]. In order to evaluate the mass-splitting in the sector of new heavy mesons $M_{q}=(q \bar{U})$, we use the relation (2) and take into account that $m(U) \approx m\left(M_{q}\right)=M$. Then, for the case of heavy meson with mass $M=10 \mathrm{TeV}$, we get:

$$
\frac{\delta m(M)}{\delta m(B)}=\frac{m\left(M^{*}\right)-m(M)}{m\left(B^{*}\right)-m(B)} \approx \frac{m_{b}}{M} \longrightarrow \delta m(M) \approx \delta m(B) \frac{m_{b}}{M} \approx 2 \mathrm{KeV}
$$

Thus, there is hyperfine mass-splitting $\delta m(M) \sim \mathrm{KeV}$ between the first excited and ground states of new heavy mesons $M_{q}$. We should note that this evaluation of mass-splitting is in accordance with approximate Expression [14]:

$$
\delta m(M) \sim \Lambda_{Q C D} \frac{\Lambda_{Q C D}(M)}{m(M)}
$$

From the Expression (4), it follows that, for $m(M) \sim 10^{4} \mathrm{GeV}, \Lambda_{Q C D}=0.218 \mathrm{GeV}$ (QCD scale) and $\Lambda_{Q C D}(M) \sim 0.1 \mathrm{GeV}$ (M-scale), the value of mass-splitting is $\delta m(M) \sim 1 \mathrm{KeV}$. The hyperfine splitting can lead to the additional mechanism of the DM cooling in the process of evolution at an early stage through the dissipative radiation of the photons which arises as a result of recombination.

The presence of excited states of hadronic DM particles provides the possibility of interaction of neutral heavy component with radiation. As was estimated above, transition to the lowest excited states of the system $M^{0}=(u \bar{U})$ can be realized by absorption of photons in $\mathrm{KeV}$ range and upper. This range is situated near the bound of $X$-rays (Roentgen) and $\gamma$-rays spectral band with the wavelength $\lambda \sim 10^{-9} \mathrm{~cm}$. Furthermore, we assume that the two-quark system $M^{0}=(u \bar{U})$ has the characteristic size an order of nucleon radius, $R_{M} \sim 10^{-13} \mathrm{~cm}$. Because $R_{M} \ll \lambda_{\text {trans }}$, interaction of $M^{0}$ with photons is caused by the high multi-pole expansion of the charge distribution in the system $(u \bar{U})$. As a result, the cross-section of $\gamma M^{0}$ scattering is rather small and these heavy mesons are, in fact, the dark matter. Here, we should note that, at $\lambda_{\text {trans }} \ll R_{M}$, that is, $E_{\gamma} \gg 10 \mathrm{MeV}$, the interaction $\gamma M^{0}$ become large and the hidden matter can not be absolutely "dark". To analyze this aspect, we need in more detail description of electromagnetic transitions in $M$-systems, which require, for instance, the building of potential model of superheavy-light two-quark systems.

\section{Interaction of New Hadrons with Standard Leptons}

Low-energy interaction of new heavy hadrons with the standard leptons can be described by the effective vertex of type $M_{a} M_{b} V$, where $M_{a}$ is $M^{0}$ or $M^{-}$and $V=Z, W$. From the quark structure of new mesons, $M=(Q q)$, one can see that the vertex $M M Z$ is stipulated by Z-boson interaction with both quarks, $Q$ and $q$. Therefore, we have to take into account interference of these sub-processes at the quark level. As was noted in the Introduction, in the scenarios under consideration, new quark $Q$ weakly interacts with $Z$-boson and does not interact with $W$-bosons. Thus, the vertex $M_{a} M_{b} W$ is generated by interaction of the $W$-boson with the standard light quark $q$ only. This vertex can be 
defined in the form-factor approach (for the exclusive process) or in the spectator approach (for the inclusive process). Decay $M^{-} \rightarrow M^{0} W^{-} \rightarrow M^{0} e^{-} \bar{v}_{e}$, where $M^{0}=(\bar{U} u)$ and $M^{-}=(\bar{U} d)$ was described in References [4,5] analogous with the standard heavy-light meson decays. Using the method of calculation in HQS approximation (see the review of Kowalski in [12]), we have derived the width of this decay in the form [4]:

$$
\left.\Gamma\left(M^{-} \rightarrow M^{0} e^{-} \bar{v}_{e}\right)=\frac{G_{F}^{2} \Delta M^{5}\left|U_{u d}\right|^{2}}{60 \pi^{3}}=\frac{g^{4}\left|U_{u d}\right|^{2}}{15 \cdot 2^{7} \pi^{3}} \frac{(\Delta M)^{5}}{M_{W}^{4}}\right),
$$

where $g$ is the constant of weak interaction, $U_{u d}$ is the element of a Kobayashi-Maskawa (CM) matrix and the mass splitting between charged and neutral mesons, $\Delta M=m\left(M^{-}\right)-m\left(M^{0}\right)$, was defined in the previous section. Furthermore, this expression we use for the determination of effective coupling constant in the vertex $M M W$. Effective Lagrangian of $W M M$ interaction in the simplest differential form is as follows:

$$
L^{e f f}(W M M)=i G_{W M} W^{+\mu}\left(\bar{M}^{0} \partial_{\mu} M^{-}-\partial_{\mu} \bar{M}^{0} M^{-}\right)+\text {h.c.. }
$$

Here, we should note that Lagrangian (6) leads to the vertex with the same structure in the momentum representation as one in the form-factor approach [4]. Using Lagrangian (6), we calculate the width $\Gamma\left(M^{-} \rightarrow M^{0} e^{-} \bar{v}_{e}\right)$ and define the effective constant $G_{W M}$ by matching the result with the expression (5). Calculating the width in approximation $m_{e}^{2} \ll(\Delta M)^{2}$, we have the following expression:

$$
\Gamma\left(M^{-} \rightarrow M^{0} e^{-} \bar{v}_{e}\right) \approx \frac{g^{2} G_{W M}^{2}}{15 \cdot 2^{4} \pi^{3}} \frac{(\Delta M)^{5}}{M_{W}^{4}} .
$$

Comparison of expressions (5) and (7) gives the definition of the effective coupling constant, $G_{W M}^{2}=g^{2}\left|U_{u d}\right|^{2} / 2^{3}$ or $G_{W M}=g U_{u d} / 2 \sqrt{2}$. We should note that the value of coupling constant $G_{W M}$ is the same as the fundamental constant in $W$-boson interaction with quark current $\bar{u} \gamma_{\mu}\left(1-\gamma_{5}\right) d$. Thus, the spectator approach at the case is valid and directly follows from the structure of sub-process at the fundamental quark level. Then, generalization of effective Lagrangian (6) including a full set of light quarks to the states of type $M_{q}=(q \bar{U})$ is straightforward:

$$
L^{e f f}(W M M)=i \frac{g U_{i k}}{2 \sqrt{2}} W^{+\mu}\left(\bar{M}_{u i} \partial_{\mu} M_{d k}-\partial_{\mu} \bar{M}_{u i} M_{d k}\right)+\text { h.c., }
$$

where $u i=u, c, t ; d k=d, s, b ; U_{i k}$ are elements of CM matrix, $M_{u i}=(u i \bar{U})$, and $M_{d k}=(d k \bar{U})$.

The structure of Lagrangian (8) describing the charge transition can be used for the description of neutral processes too. Namely, low-energy interaction of M-particles with Z-boson can be represented in the same form:

$$
L^{e f f}(Z M M)=i G_{Z M}\left(\bar{M}_{q} \partial_{\mu} M_{q}-\partial_{\mu} \bar{M}_{q} M_{q}\right) .
$$

As the effective vertexes in this case are generated by neutral currents at the quark level, the structure of $L^{e f f}(Z M M)$ preserves flavor $q i \rightarrow q i$. In contrast to (8), effective coupling $G_{Z M}$ is produced by interactions of $Z$ with both quarks: $Q$ and $q$. Thus, it is defined by coupling $g_{1} \sin \theta_{w}$ in the vertex $Z U U$ and $g_{2} c_{q} / 4 \cos \theta_{w}$ in the standard vertex $Z q q$, which are different. For the rough estimations, we can use an average value of couplings, but in the general case we meet the problem of an effective coupling definition. We should note that the electromagnetic vertex $\gamma M M$ can be represented in the form (9) for the charged M-particles only, where $G_{\gamma M}= \pm e$, provided the condition $\lambda_{\gamma}>R_{M}$ is fulfilled. In the case of neutral particle $M^{0}$, under this condition, electromagnetic contribution to the interaction is suppressed (see, also, Section 2). Effective Lagrangians (8) and (9) do not describe transitions to the excited states which were considered in Section 2. These transitions strongly complicate the structure of interaction and should be taken into account when we consider radiation in the recombination process. 
Scattering of the low-energy standard leptons on the new heavy $M$-particles is defined by the diagram with $W$-boson in the $t$-channel intermediate state. We describe this process with the help of Lagrangian (6) with coupling constant $G_{W M}=g U_{u d} / 2 \sqrt{2}$ and standard vertex $W l \bar{v}_{l}$, where $l=e, \mu, \tau$. In further calculation, we neglect the lepton mass and mass-splitting $\Delta M$ and get the following simple formula for the cross-section:

$$
\sigma\left(l^{-} M^{0} \rightarrow v_{l} M^{-}\right)=\frac{3 g^{4}\left|U_{u d}\right|^{2}}{2^{10} \pi M_{W}^{4}} s\left(1-\frac{M^{2}}{s}\right)^{2},
$$

where $\sqrt{s}$ is full energy in the center-of-mass system and $M \approx 10 \mathrm{TeV}$ [6]. For the case of non-relativistic $M$-particles, this expression can be rewritten in the form:

$$
\sigma\left(l^{-} M^{0} \rightarrow v_{l} M^{-}\right)=\frac{3 G_{F}^{2}\left|U_{u d}\right|^{2}}{8 \pi}\left(E_{l}+W\right)^{2},
$$

where $E_{l}$ is lepton energy and $W=M v^{2} / 2$ is kinetic energy of the $M$-particle. The full process of lepton scattering on $M^{0}$ is as follows: $l^{-} M^{0} \rightarrow v_{l} M^{-} \rightarrow v_{l} M^{0} e^{-} \bar{v}_{e}$. Thus, neutrinos with energy $E_{v} \sim E_{l}$ and an electron-antineutrino pair appear with total energy $E \sim \delta M$ in the final state. Due to neglect of the lepton mass, for cross-section of the process $v_{l} M^{0} \rightarrow l^{-} M^{+}$, we get the same expression.

\section{New Heavy Hadrons Interaction with Nucleons}

At the modern stage of evolution, heavy DM particles are non-relativistic with an average velocity $\sim 10^{-3}$ in co-moving frame (with respect to Galaxy). From the kinematics of the process, it follows (see Equation (19)) that low-energy nucleon interaction with DM particles can be described in the framework of effective meson-exchange theory. To do this, we need low-energy effective Lagrangian, which describes the interaction of nucleons and new hadrons with mesons in the intermediate states (meson-exchange approach). The nucleon-meson vertexes are represented in [15], where the interactions were introduced basing on the gauge scheme $U(1) \times S U(3)$. There, $U(1)$ is a semi-strong interaction group corresponding to the singlet vector meson exchange; $S U(3)$ is a group of baryon symmetry, which introduces the octet of vector mesons. It was shown in [15] that the gauge model of baryon-meson interactions satisfactorily describes the low-energy hadrons phenomenology. In particular, the gauge model predicts three mass relations in vector and scalar nonets of mesons and in an octet of baryons reasonably describes decay widths of vector mesons and the main properties of nucleon-nucleon interactions. Moreover, the gauge approach allows us to analyze baryon-meson interaction at low energy with a minimal set of parameters. A principal part of the gauge baryon-meson model is considered in Reference [16], where the set of multiplets and gauge structure are presented. The necessary part of physical Lagrangian describing the interaction of nucleons with vector mesons has a conventional form:

$$
\begin{aligned}
L_{N V} & =g_{\omega} \omega_{\mu}\left(\bar{p} \gamma^{\mu} p+\bar{n} \gamma^{\mu} n\right)+\frac{1}{2} g \rho_{\mu}^{0}\left(\bar{p} \gamma^{\mu} p-\bar{n} \gamma^{\mu} n\right) \\
& +\frac{1}{\sqrt{2}} g \rho_{\mu}^{+} \bar{p} \gamma^{\mu} n+\frac{1}{\sqrt{2}} g \rho_{\mu}^{-} \bar{n} \gamma^{\mu} p,
\end{aligned}
$$

where $g_{\omega}=\sqrt{3} g / 2 \sin \theta$ and $\theta$ is the angle of singlet-octet mixing in the sector of vector fields. Thus, the gauge realization of the meson-exchange approach contains two model parameters only, which were defined from experimental data. The model parameters fitting of vector mesons decays and the data on $\omega-\phi$ mixing give the results: $g^{2} / 4 \pi \approx 3.4$ and $\sin \theta \approx 0.78$.

The interaction of new hadrons with vector mesons can be described with help of the same gauge scheme of $S U(3)$ symmetry dynamic realization. To do this, we should extend the doublet of new heavy mesons $M=\left(M^{0}, M^{-}\right)$to the triplet, which is the fundamental representation of the $S U(3)$ group. We fulfill this extension by the including of a standard s-quark as a part of new 
mesons: $M=\left(M^{0}, M^{-}, M_{s}^{-}\right)=\left(M_{u}, M_{d}, M_{s}\right)$, where $M^{0}=M_{u}=(\bar{U} u), M^{-}=M_{d}=(\bar{U} d)$ and $M_{s}^{-}=M_{s}=\left(\bar{U}_{s}\right)$. We should note that $S U(2)$ isotopic symmetry of nucleons consisting of $u$ - and $d$-quarks is nearly exact. The extension of the isotopic symmetry to $S U(3)$ group is an approximation; however, it allows us to use the symmetry of baryon octet which consists of $u, d$, and $s$ quarks. Essential violation of $S U(3)$-symmetry in the sector of usual hadrons is stipulated by the large value of mass-splitting of $s$ - and $d$-quarks. In the case under consideration, heavy hadrons $M_{q}=(q \bar{U})$ consist of new heavy $(U)$ and standard light quarks $(u, d, s)$, so the mass-splitting in the sector of $M_{q}$-particles is negligible. Thus, the symmetry $S U(3)$ is almost exact, and we can apply its dynamic (gauge) realization with the same vector (gauge) mesons.

Effective low-energy Lagrangian of $M$-particles interactions with vector singlet $V_{\mu}^{0}$ and octet $V_{\mu}$ has conventional gauge form:

$$
L_{M V}=\left(D^{\mu} M\right)^{\dagger} D_{\mu} M, \quad D_{\mu} M=\left(\partial_{\mu}-i t V_{\mu}^{0}-\frac{i g}{\sqrt{2}} V_{\mu}\right) M,
$$

where $(A)^{\dagger}$ is Hermitian conjugation, $V_{\mu}=V_{\mu}^{a} \tau_{a} / 2$, and $t$ is a new model parameter. Physical Lagrangian of $M$-particles interaction with vector mesons directly follows from (13):

$$
\begin{aligned}
L_{M V} & =i G_{\omega M} \omega^{\mu}\left(\bar{M}^{0} M_{, \mu}^{0}-\bar{M}_{, \mu}^{0} M^{0}+M_{, \mu}^{+} M^{-}-M^{+} M_{, \mu}^{-}\right) \\
& +\left(i G_{\omega M_{s}} \omega_{\mu}+i G_{\phi M_{s}} \phi_{\mu}\right)\left(M_{s}^{+} M_{s}^{-, \mu}-M_{s}^{+, \mu} M_{s}^{-}\right) \\
& +\left(\frac{i g}{2} \rho_{\mu}^{0}+i G_{\phi M} \phi_{\mu}\right)\left(\bar{M}^{0} M_{, \mu}^{0}-\bar{M}_{, \mu}^{0} M^{0}+M_{, \mu}^{+} M^{-}-M^{+} M_{, \mu}^{-}\right) \\
& +\frac{i g}{\sqrt{2}} \rho^{+\mu}\left(\bar{M}^{0} M_{, \mu}^{-}-\bar{M}_{, \mu}^{0} M^{-}\right)+\frac{i g}{\sqrt{2}} \rho^{-\mu}\left(M^{+} M_{, \mu}^{0}-M_{, \mu}^{+} M^{0}\right) .
\end{aligned}
$$

In Equation (14), the coupling constants $G_{a}$ are some functions of the model parameters $t, g$ and $\cos \theta$ which will be determined below. To define these constants, we take into consideration quark content of the meson $\phi=(s \bar{s})$ and new heavy mesons $M^{0}=(\bar{U} u), M^{-}=(\bar{U} d)$. From the quark structure of corresponding vertexes, it follows that $G_{\phi M}=0$ and we get following relation:

$$
G_{\phi M}=t \sin \theta-\frac{g}{2 \sqrt{3}} \cos \theta=0 \Longrightarrow t=\frac{g}{2 \sqrt{3}} \cot \theta
$$

From the quark content of the vector mesom $\omega$ and new meson $M_{s}^{-}=(\bar{U} s)$, the equality $G_{\omega M_{s}}=0$ and relation follows:

$$
G_{\omega M_{s}}=\frac{g}{2 \sqrt{3} \sin \theta}\left(1-3 \sin ^{2} \theta\right)=0 \Longrightarrow \sin \theta=\frac{1}{\sqrt{3}} .
$$

With the help of Equations (15) and (16), we can represent the remaining coupling constants by the expressions:

$$
G_{\omega M}=\frac{g}{2 \sqrt{3} \sin \theta}=\frac{g_{\omega}}{3}, G_{\phi M_{s}}=-\frac{\sqrt{3}}{2} g \cos \theta .
$$

Three-linear interactions of new heavy mesons with usual pseudoscalar mesons are absent because the vertex $\varphi M M$ is forbidden due to parity conservation. This is an important feature differing new hadrons from the nucleon, for which the vertex $\varphi N N$ is permitted. Thus, the interaction of nucleons with $M$-particles can not be realized through the one-pion exchange at the tree level.

Scattering of ordinary nucleons on new heavy mesons at low energy is described by $t$-channel diagrams with usual vector and scalar mesons in the intermediate states. As was noted above, the diagrams with an intermediate pseudoscalar mesons ( $\pi$-meson, for example) are forbidden at the tree level by parity conservation. Such interaction can be realized by two-pion exchange due to the 
vertex $M M \varphi \varphi$ is permitted. However, the vertex $N N \varphi \varphi$ is absent, so two-pion exchange is possible in the model at the loop level only.

Now, we consider the kinematics of elastic scattering process $M N \rightarrow M N$, where $M=\left(M^{0}, M^{-}\right)$ and $N=(p, n)$. For the case of non-relativistc particles, the value of momentum transfer $Q^{2}=-q^{2}$ is described by the expression:

$$
Q^{2}=-\left(P_{N}-K_{N}\right)^{2} \approx k_{N}^{2}+p_{N}^{2}-2 \cos \theta_{s c} k_{N} p_{N}
$$

where $p_{N}$ and $k_{N}$ are absolute values of nucleon's three-momenta in the initial and final states, respectively, and $\theta_{S c}$ is the angle of scattering. In the center-of-mass system (CMS) $v_{M} m_{M}=v_{N} m_{N}$, where $v_{M}+v_{N}=v_{r}$ is a relative velocity. In the galaxy, the rest-system (comoving system) velocity of the DM particle $v \sim 10^{-3}$ and the velocity of non-relativistic nucleons have the same order of magnitude, $v_{r} \sim 10^{-3}$. Thus, we get the relations:

$$
v_{N}=v_{r} /\left(1+\frac{m_{N}}{m_{M}}\right) \sim v ; v_{M}=v_{r} /\left(1+\frac{m_{M}}{m_{N}}\right) \sim v \frac{m_{N}}{m_{M}}
$$

Thus, in CMS, $p_{N}=p_{M} \approx m_{N} v_{r}$ and $k_{N} \approx k_{M} \approx m_{N} v_{r}$. From Equation (18), it follows that the maximal value of momentum transfer is $Q_{\text {max }}^{2}=(p k)^{2} \approx 4 m_{N}^{2} v_{r}^{2}$ that is $Q_{\max } \approx m_{N} v_{r} \sim 10^{-3} m_{N}$. Thus, the momentum transfer is much less than the mass of intermediate mesons $\left(m \sim m_{N}\right)$, and the meson-exchange model is relevant.

The process of elastic scattering of type $N M \rightarrow N M$ is described by the $t$-channel diagrams with vector $v=\left(\omega, \rho^{0}\right)$ and scalar $s=\left(f_{0}, a_{0}^{0}\right)$ meson exchange. The ratio of scalar and vector contributions into the amplitude squared is:

$$
\frac{A_{s}^{2}}{A_{v}^{2}} \sim \frac{g_{s p}^{2} g_{s M}^{2}}{m_{M}^{2}} ; g_{s p}=k v_{1} \sim 0.1 m_{N},
$$

where $g_{s p}$ was defined in References $[7,15]$ and $g_{s M}$ is new dimensionless coupling constant. Taking the realistic value $g_{s M} \sim 1$, the ratio of contributions is as follows:

$$
\frac{A_{s}^{2}}{A_{v}^{2}} \sim 10^{-2} \frac{m_{N}^{2}}{m_{M}^{2}} \sim 10^{-10}
$$

From this estimation, the effect of strong suppression of the scalar meson exchange contribution follows. Using the above expressions for the model Lagrangian, we derived the expression for the cross-section of the elastic scattering process $N_{a} M_{b} \rightarrow N_{a} M_{b}$ :

$$
\sigma\left(N_{a} M_{b} \rightarrow N_{a} M_{b}\right)=\frac{g^{4} m_{p}^{2}}{16 \pi m_{v}^{4}}\left(1+\frac{k_{a b}}{\sin ^{2} \theta}\right)^{2}
$$

where $N_{a}=(p, n), M_{b}=\left(M^{0}, M^{-}\right), g^{2} / 4 \pi \approx 3.4, \sin \theta=1 / \sqrt{3}$ and $k_{a b}= \pm 1$ for the case of $p$ or $n$. Here, we used an approximate relations $m_{p} \approx m_{n}, m\left(M^{0}\right) \approx m\left(M^{-}\right)$and $m_{v}=m_{\rho} \approx m_{\omega}$. As a result, we get a rather large value of cross-section, for example $\sigma\left(p M^{0} \rightarrow p M^{0}\right) \approx 0.9$ barn, and this process can be detected as nucleon recoil in solid detectors. However, the value of kinetic cross-section $(\sigma v)$ is suppressed by the factor $v_{r} \sim 10^{-3}$. Note that the cross-section for the nonrelativistic scattering does not depend on the mass $m_{M}$ and the angle of scattering. A large cross-section of elastic scattering of nucleon on hadronic DM can stipulate appreciable mutual exchange of momentum between DM halo and galaxy during their evolution. The problem of the connection between galaxies and their DM halo can play an important role in modern understanding of galaxy formation [17].

Furthermore, we consider non-elastic scattering of type $N_{a} M_{b} \rightarrow N_{c} M_{d}$, where $N_{a}=(p, n)$ and $M_{b}=\left(M^{0}, M^{-}\right)$. Some of these processes have a threshold, and, because the mass splitting in both doublets, nucleons, and new hadrons, is very small, kinematics of these processes (far from the 
threshold) is almost the same as the elastic scattering one. In particular, the value of momentum transfer is defined by Equation (18) and $Q_{\max } \approx m_{N} v_{r} \sim 10^{-3} m_{N}$. In the case under consideration, the dominant contribution follows from the $t$-channel diagram with $\rho^{ \pm}$in the intermediate state. The cross-section can be represented in a form which describes the presence or absence of threshold explicitly:

$$
\sigma\left(N_{a} M_{b} \rightarrow N_{c} M_{d}\right)=\frac{g^{4} m}{8 \pi v_{r} m_{v}^{4}} \sqrt{2 m}\left[E_{a}-\Delta_{a b}\right]^{1 / 2},
$$

where $E_{a} \approx m_{a} v_{r}^{2} / 2, m\left(N_{a}\right)=m_{a} \approx m_{b} \approx m, \Delta_{a b}$ is the combination of $\Delta M=m\left(M^{+}\right)-m\left(M^{0}\right)$ and $\Delta m=m_{n}-m_{p} \approx 1.4 \mathrm{MeV}$ (see the Table 1). Expression (23) can be rewritten in a more simple form:

$$
\sigma\left(N_{a} M_{b} \rightarrow N_{c} M_{d}\right)=\frac{g^{4} m^{2}}{8 \pi m_{v}^{4}}\left[1-\frac{\Delta_{a b}}{E_{p}}\right]^{1 / 2} .
$$

From (24), it follows that the reactions have threshold $E_{p}^{t h r}=\Delta_{a b}$ when $\Delta_{a b}>0$.

In Table 1, these characteristics are shown for the case of four basic reactions, $p M^{0} \rightarrow n M^{+}$, $n M^{+} \rightarrow p M^{0}, n M^{0} \rightarrow p M^{-}$and $p M^{-} \rightarrow n M^{0}$.

Table 1. The threshold parameters $\Delta_{a b}$.

\begin{tabular}{lll}
\hline$N_{a} M_{b} \rightarrow N_{c} M_{d}$ & $\Delta_{a b}=f(\Delta M, \Delta m)$ & signum $\Delta_{a b}$ \\
\hline$p M^{0} \rightarrow n M^{+}$ & $\Delta_{p 0}=\Delta M+\Delta m$ & $\Delta_{p 0}>0$ (threshold) \\
\hline$n M^{+} \rightarrow p M^{0}$ & $\Delta_{n+}=-\Delta M-\Delta m$ & $\Delta_{n+}<0$ (non-threshold) \\
\hline$n M^{0} \rightarrow p M^{-}$ & $\Delta_{n 0}=\Delta M-\Delta m$ & $\Delta_{n 0}>0(\Delta M>\Delta m)$ \\
\hline$p M^{-} \rightarrow n M^{0}$ & $\Delta_{p-}=-\Delta M+\Delta m$ & $\Delta_{p-}>0(\Delta M<\Delta m)$ \\
\hline
\end{tabular}

Let us consider the first reaction, $p M^{0} \rightarrow n M^{+}$, where $E_{p} \approx m_{p} v_{r}^{2} / 2, \Delta M=m\left(M^{+}\right)-m\left(M^{0}\right)$ and $\Delta m=m_{n}-m_{p} \approx 1.4 \mathrm{MeV}$. From the threshold energy $E_{p}^{t h r}=\Delta M+\Delta m \equiv \Delta_{p 0}$, it follows the value of the threshold relative velocity $v_{r}^{t h r}=\sqrt{2 \Delta_{p 0} / m_{p}}$. For the realistic case $\Delta_{p 0}=10 \mathrm{MeV}$, we get rather large velocity $v_{r}^{\text {thr }}=0.1$, which is much greater than the DM particles' velocity at the moment, $v_{r} \sim 10^{-3}$. Thus, the process is not going now and can not be registered by charged particle detectors. The second reaction is the non-threshold one with unstable but long-lived particles in the initial state. The third and fourth reactions can be threshold or non-threshold depending on the value of $\Delta M / \Delta m$. The first, third, and fourth reactions lead to the final states with the unstable particles; they go in two stages, for instance, $p M^{0} \rightarrow n M^{+} \rightarrow p e^{-} \bar{v}_{e} M^{0} e^{-} \bar{v}_{e}$ and $n M^{0} \rightarrow p M^{-} \rightarrow p M^{0} e^{-} \bar{v}_{e}$. Here, we should note that the third reaction, $n M^{0} \rightarrow p M^{-} \rightarrow p M^{0} e^{-} \bar{v}_{e}$, may be the most interesting one due to the presence of metastable charge heavy particle $M^{-}$, which can be registered in atmosphere. Note that some indirect evidence of such particles presence was reported in [7] (and references therein), where experimental and theoretical premises of new stable hadron existence were considered. Thus, we can have a rich low-energy phenomenology of nucleon-DM scattering with specific signature of the final states.

The processes of $M^{0} \bar{M}^{0}$-annihilation go through both strong and electro-weak channels with ratio of probability an order of value $\left(\alpha / \alpha_{s}\right)^{2} \sim 10^{-2}$. We have no the theory of high-energy strong interaction of $M$-particles; however, the process of annihilation can be approximately described at the sub-process level, namely $Q \bar{Q} \rightarrow q \bar{q}, g g \rightarrow$ jets, where $q$ is standard light quark and $g$ is gluon. Using this approach, we estimated the cross-section of annihilation and noted the peculiarity of description of full annihilation process [5,6]. Here, we should note that the main products of this process in the final state are the pairs of stable standard particles, mainly $p \bar{p}$ and small fraction of $e^{+} e^{-}, v \bar{v}, 2 \gamma$ with total energy $E_{t o t} \approx 2 M$. 


\section{Discussion}

Composite particles, such as atom or hadron (meson or baryon), always have excited states with are split in energy. The value of this splitting is defined by the corresponding interaction (electro-magnetic or strong). We considered the main structural features and interaction properties of new heavy hadrons proposed as dark matter candidates. Excited states of new hadrons have been considered analogously with the standard heavy-light mesons case. We show that there exists the hyperfine structure of excited levels which can lead to electromagnetic transitions and recombination in the processes of new hadrons interaction with the ordinary matter and cosmic rays. Interaction of hadronic DM with photons gives rise to the problem of separation of the terms "dark matter" and "hidden matter". It was noted that this problem becomes real when discussing the gamma-ray spectrum induced by the DM inhomogeneities.

Considerating the DM interactions with the ordinary particles, leptons and protons, it is important to analyze possible signals which are produced in these processes. To describe the hadronic DM interaction with leptons through $W$-boson, the necessary effective vertex, $W M M$, was constructed. The $W M M$ vertex is caused by $W$-boson interaction with the standard light quark, $q$, only. Effective coupling constant of WMM-interaction had been defined from the width of decay $M^{-} \rightarrow M^{0} W^{-} \rightarrow$ $M^{0} e^{-} \bar{v}_{e}$ in the form-factor approach. Knowing this vertex, we calculated the cross-section of the lepton scattering on new heavy hadrons. From the quark content of $M$-mesons, we can conclude that $Z M M$ vertex is generated by interaction of $Z$-bosons with both quarks, $Q$ and $q$.

Thus, to describe the dark matter signals in hadronic processes, we have developed and analyzed the low-energy effective model of new hadrons interaction with ordinary hadrons. The DM-nucleon low-energy interaction is considered in the framework of meson-exchange approach and realized as the gauge model with the $S U(3)$-symmetry using. In this model, we have derived analytical expressions for the cross-sections of elastic and inelastic collisions of nucleons and new hadrons. These results can be used for the analysis of the DM interactions with interstellar gas in the Galaxy and with the Earth atmosphere. The most important manifestation of such processes can be the emerging of metastable heavy charged particles, $M^{-}$. They can be registered by ground detectors due to characteristic radiation of soft leptons (when the charged heavy hadron decays into the neutral one) or as specific EAS (Extended Air Shower) produced by heavy charged hadron. Thus, the hadron scenario of the DM suggests considering some new aspect of connection between galaxies and their DM halos; it can also stipulate some peculiarities of galaxy formation.

\section{Data Availability}

The graphic data used to support the findings of this study are available from the corresponding author upon request.

Author Contributions: Conceptualization, V.K. and V.B. All authors have read and agreed to the published version of the manuscript.

Funding: Research was financially supported by Southern Federal University, 2020 (Ministry of Science and Higher Education of the Russian Federation) (In Gr/2020-03-IF).

Acknowledgments: The authors are sincerely grateful to M. Yu. Khlopov for fruitful cooperation during the scientific research.

Conflicts of Interest: The authors declare no conflict of interest.

\section{References}

1. Aprile, E.; Aalbers, J.; Agostini, F.; Alfonsi, M.; Amaro, F.D.; Anthony, M.; Arneodo, F.; Barrow, P.; Baudis, L.; Bauermeister, B.; et al. First, Dark Matter Search Results from the XENON1 Experiment. Phys. Rev. Lett. 2017, 119, 181301. [CrossRef] [PubMed]

2. Huo, R.; Matsumoto, S.; Sming Tsai, Y.L.; Yanagida, T.T. A scenario of heavy but visible baryonic dark matter. J. High Energy Phys. 2016, 1609, 162-166. [CrossRef] 
3. Luca, V.; Mitridate, A.; Redi, M.; Smirnov, J.; Strumia, A. Colored Dark Matter. Phys. Rev. D 2018, 97, 115024. [CrossRef]

4. Beylin, V.; Kuksa, V. Dark Matter in the Standard Model Extension with Singlet Quark. Adv. High Energy Phys. 2018, 18, 8670954. [CrossRef]

5. Beylin, V.; Kuksa, V. Possibility of hadronic dark matter. Int. J. Mod. Phys. D 2019, 28, 1941001. [CrossRef]

6. Beylin, V.; Khlopov, M.; Kuksa, V.; Volchanskiy, N. Hadronic and Hadron-Like Physics of Dark Matter. Symmetry 2019, 11, 587. [CrossRef]

7. Bazhutov, Y.N.; Vereshkov, G.M.; Kuksa, V.I. Experimental and Theoretical Premises of New Stable Hadron Existence. Int. J. Mod. Phys. A 2017, 2, 1759188. [CrossRef]

8. Khlopov, M.Y. Modern Physics of dark matter im the light of dark atoms. Phys. Lett. A 2011, 26, $2823-2839$.

9. Khlopov, M.Y. Introduction to the special issue on indirect dark matter searches. Int. J. Mod. Phys. A 2014, 29, 1443002. [CrossRef]

10. Cudell, J.R.; Khlopov, M. Dark atoms with nuclear shell: A status review. Int. J. Mod. Phys. D 2015, 24, 1545007. [CrossRef]

11. Ebert, D.; Galkin, V.O.; Faustov, R.N. Mass spectrum of orbitally and radially excited heavy-light mesons in the relativistic quark model. Phys. Rev. D 1998, 57, 5663-5669. [CrossRef]

12. Tanabashi, M.; Hagiwara, K.; Hikasa, K.; Nakamura, K.; Sumino, Y.; Takahashi, F.; Tanaka, J.; Agashe, K.; Aielli, G.; Amsler, C.; et al. The Review of Particle Physics. Phys. Rev. D 2018, 98, 1. [CrossRef]

13. Mutuk, H. Mass Spectra and Decay Constants of Heavy-Light Mesons: A Case Study of QCD Sum Rules and Quark Model. Adv. High Energy Phys. 2018, 2018, 8095653. [CrossRef]

14. Grozin, A.G. Lectures on perturbative HQET 1. arXiv 2000, arXiv:hep-ph/0008300.

15. Vereshkov, G.M.; Kuksa, V.I. U(1)SU(3)-gauge model of baryon-meson interactions. Yad. Fiz. 1991, 54, 1700.

16. Beylin, V.A.; Kuksa, V.I. Interaction of Hadronic Dark Matter with Nucleons and Leptons. Symmetry 2020, 12, 567. [CrossRef]

17. Wechsler, R.H.; Tinker, J.L. The Connection between Galaxies and their Dark Matter Halos. Annu. Rev. Astron. Astrophys. 2018, AA, 1-56.

(C) 2020 by the authors. Licensee MDPI, Basel, Switzerland. This article is an open access article distributed under the terms and conditions of the Creative Commons Attribution (CC BY) license (http:/ / creativecommons.org/licenses/by/4.0/). 